\title{
Clinical Significance of Serum p53 Antibody in the Early Detection and Poor Prognosis of Gastric Cancer
}

\author{
MASAKI KUNIZAKI ${ }^{1}$, AKIKO FUKUDA ${ }^{1}$, KOUKI WAKATA $^{1}$, TETSURO TOMINAGA ${ }^{1}$, \\ TAKASHI NONAKA ${ }^{1}$, TAKURO MIYAZAKI ${ }^{1}$, KEITARO MATSUMOTO $^{1}$, \\ YORIHISA SUMIDA ${ }^{1}$, SHIGEKAZU HIDAKA ${ }^{1}$, TORU YASUTAKE ${ }^{1}$, TERUMITSU SAWAI $^{1}$, \\ RYUJI HAMAMOTO ${ }^{2}$, ATSUSHI NANASHIMA ${ }^{1}$ and TAKESHI NAGAYASU ${ }^{1}$ \\ ${ }^{1}$ Division of Surgical Oncology, Nagasaki University Hospital, Nagasaki, Japan; \\ ${ }^{2}$ Division of Molecular Modification and Cancer Biology, National Cancer Center Research Institute, Tokyo, Japan
}

\begin{abstract}
Background: The aim of this retrospective study was to evaluate the clinical relevance of serum $p 53$ antibody $(S-p 53 A b)$ as a biomarker and to investigate whether its diagnostic value could be improved when combined with other biomarkers of gastric cancer $(G C)$. Patients and Methods: Serum samples were obtained preoperatively from 208 patients with histologically-confirmed GC, including 126 stage I patients (60.6\%). Levels of S-p53Ab were assessed by a commercial laboratory using an anti-p53 detection kit. The cut-off value for $S-p 53 \mathrm{Ab}$ was $1.3 \mathrm{U} / \mathrm{ml}$. Results: S-p53Ab was detected in 16.3\% (34 of 208) of patients with GC, including 13.6\% (22 of 162) of patients with early-stage GC. The positive rates for $S$-p53Ab, carbohydrate antigen 19-9 (CA19-9), and carcinoembryonic antigen (CEA) of patients with stage I GC were $10.3 \%$ (13/126), 2.4\% (3/126), and 8.7\% (11/126), respectively. Positivity for S-p53Ab was not associated with CA19-9 or CEA positivity ( $p=0.098$ and 0.053 , respectively). The positive rate for a diagnosis of GC increased from $16.3 \%$ to $29.3 \%$ when S-p53-Ab was combined with CEA in this study. We found no significant correlation between the presence of $S-p 53 A b$ in $G C$ and overall survival. Conversely, Cox regression analysis revealed that a high level of CA19-9 was an independent prognostic factor for $G C$ in this series (hazard $\operatorname{ratio}(H R)=3.864 ; 95 \%$ confidence interval $(C I)=$ 1.248-11.959; $\quad p=0.019)$. Kaplan-Meier analyses demonstrated significant differences in survival between patients with elevated levels of both S-p53Ab and CEA and
\end{abstract}

Correspondence to: Masaki Kunizaki, Division of Surgical Oncology, Department of Translational Medical Sciences, Nagasaki University Graduate School of Biomedical Sciences, 1-7-1 Sakamoto, Nagasaki 852-8501, Japan. Tel: 095 8197304, Fax: 095 8197306, e-mail: makuni49@nagasaki-u.ac.jp

Key Words: Gastric cancer, tumor markers, serum p53 antibody. those with elevated levels of only one or neither. Conclusion: The diagnostic rate of $S-p 53 A b$ was better than that of CA19-9 and CEA in patients with stage I GC. Combined detection of $S-p 53 A b$ and CEA may improve the diagnostic sensitivity and may permit more accurate stratification of GC patients.

Gastric cancer (GC) is one of the most common cancers in the digestive tract and is the second most frequent cause of mortality worldwide (1). Several therapeutic options have been developed for GC including laparoscopic surgery, chemotherapy, and endoscopic resection including endoscopic submucosal dissection. Along with the development of endoscopic equipment, endoscopic submucosal dissection has become widely used to treat earlystage GC. On the other hand, radical surgery with regional lymph node dissection has been the mainstay of treatment for advanced GC. Although improvements have been made in early detection and surgical technologies, advanced GC is still associated with a poor prognosis. Thus, continuing interest exists in new and useful biological serum markers that will permit more accurate stratification of GC patients, improve clinical decision-making, and possibly contribute to more rational study design and analysis.

Circulating p53 antibodies in patients have been reported for various types of carcinomas, including GC (2-9). Several studies have demonstrated that serum p53 antibodies (S$\mathrm{p} 53 \mathrm{Ab}$ ) are early markers of malignant disease, a marker for monitoring patients with malignant tumors during treatment, and a prognostic factor for patients with several types of tumors (10-12). Although previous studies have attempted to evaluate the clinical value of $\mathrm{S}-\mathrm{p} 53 \mathrm{Ab}$ in different conditions, the role of $\mathrm{S}-\mathrm{p} 53 \mathrm{Ab}$ in patients with GC has not been clearly established. In the present study, we evaluated the clinical relevance of S-p53Ab and investigated whether its diagnostic value could be improved when combined with other biomarkers. 


\section{Materials and Methods}

Patients. We retrospectively reviewed a database of 208 patients with primary GC who had undergone elective surgery for GC that was performed by the same trained surgical team at the Division of Surgical Oncology, Nagasaki University Hospital (Japan) between August 2008 and December 2014. Written informed consent was obtained from each patient. The current study was approved by the Ethics Review Board (number 09062633) of Nagasaki University Hospital and complied with the standards of the Declaration of Helsinki. Patients who had undergone preoperative radiotherapy or endoscopic resection, those who had received chemotherapy, and those who died within 30 days after surgery were excluded from this study.

Serum and tumor samples. GC staging was performed according to the International Union Against Cancer (UICC7)/TNM classification (13) based on histopathological examination of specimens that had been resected to the extent possible. Serum samples were collected from each patient before treatment and stored at $-80^{\circ} \mathrm{C}$ until the assay.

Enzyme-linked immunosorbent assay (ELISA) for S-p53Ab. The Sp53Ab levels were assessed by analyzing preoperative serum samples and an anti-p53 detection kit (MESACUP anti-p53 Test; Medical and Biological Laboratories (MBL), Nagoya, Japan). In brief, serum samples were incubated for $1 \mathrm{~h}$ at $37^{\circ} \mathrm{C}$ in microtiter wells coated with wild-type human $\mathrm{p} 53$ protein or a control protein to detect nonspecific interactions. The wells were washed and then a peroxidase-conjugated goat antihuman immunoglobulin $\mathrm{G}$ that binds $\mathrm{p} 53 \mathrm{Ab}$ was applied for $1 \mathrm{hr}$ at $37^{\circ} \mathrm{C}$ followed by substrate solution for $30 \mathrm{~min}$ at $37^{\circ} \mathrm{C}$. After addition of stop solution, color development was assessed by measuring absorption at $450 \mathrm{~nm}$ using a spectrophotometer. Levels of S-p53Ab were determined from a calibration curve constructed from the specific signals of standards. $\mathrm{S}$-p53Ab levels $\geq 1.3 \mathrm{U} / \mathrm{ml}$ were considered as positive, according to the manufacturer's instructions (12).

Values for Glasgow prognostic score (GPS), carcinoembryonic antigen (CEA), and carbohydrate antigen 19-9 (CA19-9). Routine laboratory measurements including albumin, serum C-reactive protein, and tumor markers such as CEA and CA19-9 were performed on the same day to exclude any inflammatory effects of sequential preoperative procedures such as esophagogastroduodenoscopy or barium enema. According to the manufacturers, cutoff values for serum CEA and CA19-9 are $5.0 \mathrm{ng} / \mathrm{ml}$ and $37 \mathrm{U} / \mathrm{ml}$, respectively. The GPS was estimated as described previously $(14,15)$. Briefly, patients with both elevated C-reactive protein $(>1.0 \mathrm{U} / \mathrm{ml})$ and hypoalbuminemia $(<3.5 \mathrm{~g} / \mathrm{dl})$ levels were assigned a score of 2. Patients with only one of these biochemical abnormalities were assigned a score of 1 . Patients with neither of these abnormalities were assigned a score of 0 .

Statistical analysis. Statistical analyses were performed using the significance of differences among groups, which were determined using JMP ${ }^{\circledR} 10$ software (SAS Institute, Cary, NC, USA). The significance level was set at $p<0.05$. Differences among groups were calculated using the $\chi^{2}$ test or Fisher's exact test. The survival probabilities were determined using Kaplan-Meier analysis, and the significance of differences was analyzed using the log-rank test. Multivariate analysis was performed using the Cox regression model.
Table I. Relationships between clinicopathological characteristics and serum $p 53$ antibody (S-p53Ab) in patients with gastric cancer.

\begin{tabular}{|c|c|c|c|}
\hline \multirow[b]{2}{*}{ Variables } & \multicolumn{2}{|c|}{ S-p53Ab $(n=208)$} & \multirow[b]{2}{*}{$p$-Value } \\
\hline & $\begin{array}{c}\text { Positive } \\
n=34\end{array}$ & $\begin{array}{c}\text { Negative } \\
\mathrm{n}=174\end{array}$ & \\
\hline Gender (male/female) & $26 / 8$ & $116 / 58$ & 0.357 \\
\hline Age $<65 / \geq 65$ years & $15 / 19$ & $73 / 101$ & 0.815 \\
\hline Tumor site (DLM/UE) & $13 / 11 / 10$ & $70 / 56 / 48$ & 0.969 \\
\hline Histological type (diff/undiff) & $18 / 16$ & $87 / 87$ & 0.754 \\
\hline Tumor depth pT1-2/T3-4 & $19 / 15$ & $122 / 52$ & 0.104 \\
\hline TNM stage $0-\mathrm{II} / \mathrm{III}-\mathrm{IV}$ & $22 / 12$ & $140 / 34$ & 0.043 \\
\hline Lympatic invasion $0 / 1,2,3$ & $10 / 24$ & $83 / 91$ & 0.050 \\
\hline Venous invasion $0 / 1,2,3$ & $12 / 22$ & $83 / 91$ & 0.184 \\
\hline Nodal stage N0/N1-3 & $16 / 18$ & $123 / 51$ & 0.007 \\
\hline \multicolumn{4}{|l|}{ Total number of metastatic } \\
\hline lymph nodes $0-9 / 10-34$ & $21 / 13$ & $159 / 15$ & $<0.01$ \\
\hline \multicolumn{4}{|l|}{ Total number of dissected } \\
\hline lymph nodes $0-39 / 40-97$ & $12 / 22$ & $79 / 95$ & 0.277 \\
\hline \multicolumn{4}{|l|}{ Lymph node dissection (D) } \\
\hline $\mathrm{D} 0,1 / \mathrm{D} 1+, \mathrm{D} 2$ & $2 / 32$ & $7 / 167$ & 0.979 \\
\hline Surgery Laparoscopic/Open & $16 / 18$ & $124 / 50$ & 0.006 \\
\hline C-reactive protein $<1.0 / \geq 1.0 \mathrm{U} / \mathrm{ml}$ & $30 / 4$ & $166 / 8$ & 0.216 \\
\hline Albumin $<3.5 / \geq 3.5 \mathrm{~g} / \mathrm{dl}$ & $1 / 33$ & $10 / 164$ & 0.803 \\
\hline GPS: $0 / 1,2$ & $30 / 4$ & $8 / 166$ & $<0.01$ \\
\hline CEA: $<5 / \geq 5 \mathrm{ng} / \mathrm{ml}$ & $24 / 10$ & $147 / 27$ & 0.053 \\
\hline CA19-9: $<37 / \geq 37 \mathrm{U} / \mathrm{ml}$ & $29 / 5$ & $165 / 9$ & 0.098 \\
\hline
\end{tabular}

D, Deuodenum; LMN, the lower(L), middle (M), and upper (U) parts of stomach; E, esophagus; GPS, Glasgow prognostic score; S-p53Ab, serum p53 antibody; CEA, carcinoembryonic antigen; CA19-9, carbohydrate antigen 19-9.

\section{Results}

Association between the presence of $S-p 53 A b$ and clinicopathological features in patients with GC. All 208 patients (142 men (68.3\%), 66 women (31.7\%); median age $=65.8$ years) underwent gastric resection for various clinical stages of GC. Serum samples from $34(16.3 \%)$ of 208 patients with GC were positive for S-p53Ab. The mean age in this group was 66.0 years (range $=28-83$ years), and the male-to-female ratio was 3.3:1. The remaining 174 patients $(83.7 \%)$ were negative for S-p53Ab and had a mean age of 65.7 years (range $=33-85$ years) and a male-to-female ratio of $2: 1$. We analyzed clinicopathological features of these patients stratified by the presence of S-p53Ab. The positive rate of $\mathrm{S}-\mathrm{p} 53 \mathrm{Ab}$ did not differ significantly according to factors such as sex, age, tumor site, histological type, tumor depth, venous invasion, total number of dissected lymph nodes, lymph node dissection, C-reactive protein, albumin, CEA, and CA19-9. On the other hand, lymph node metastasis was found in 18 (52.9\%) of the 34 patients with a positive S-p53Ab test result but in only 51 $(29.3 \%)$ of the 174 patients with a negative S-p53Ab test 


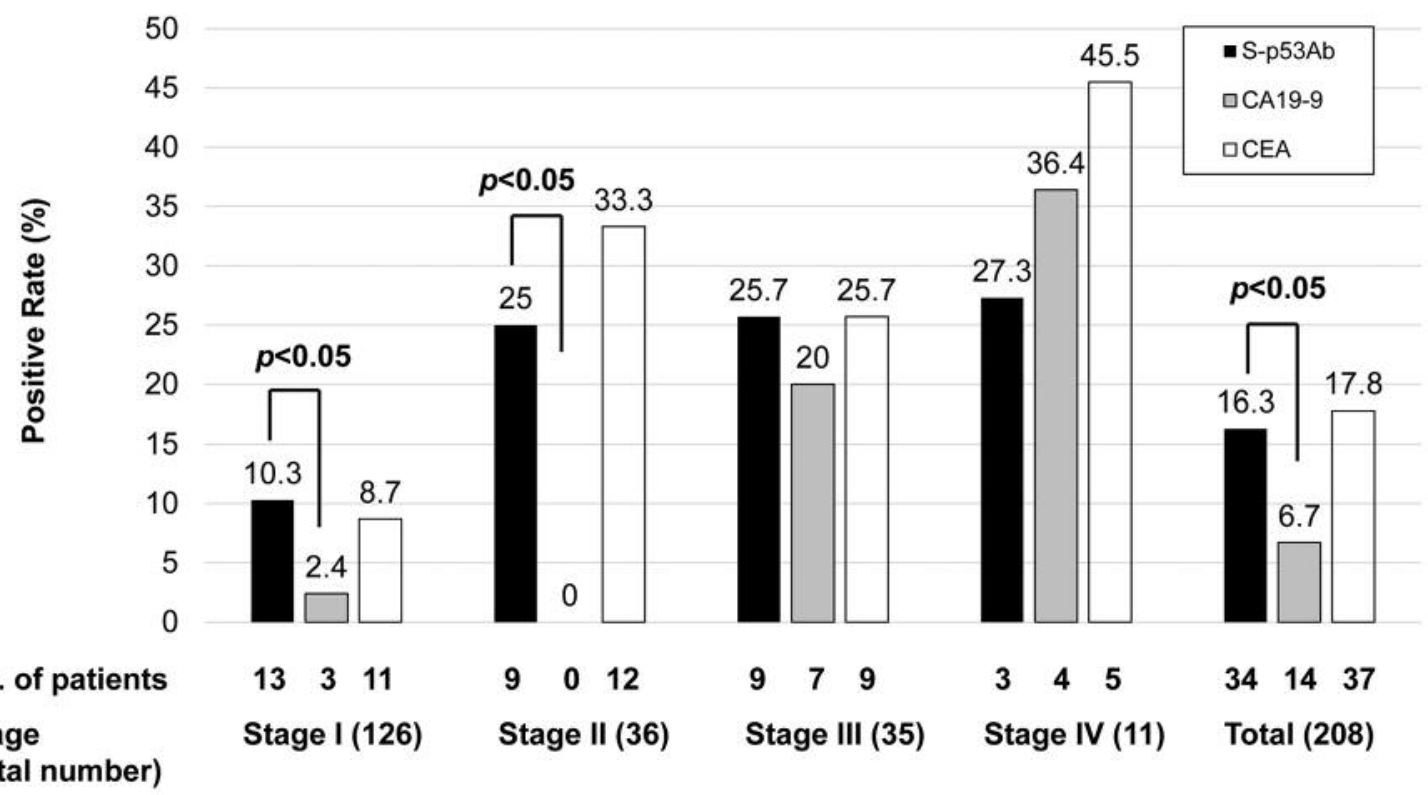

Figure 1. Correlations among the sensitivity of serum p53 antibody (S-p53Ab), carcinoembryonic antigen (CEA), and carbohydrate antigen 19-9 (CA19-9) according to clinical stage in patients with gastric cancer.

result $(p=0.007)$. Positive lymphatic invasion was also found in $24(70.6 \%)$ of the 34 patients with a positive S-p53Ab test result but in only $91(52.3 \%)$ of the 174 patients with a negative S-p53Ab test result $(p=0.049)$ (Table I).

Sensitivity of S-p53Ab, CA19-9, and CEA in patients with GC. The clinical stage was I in $126(60.6 \%)$ patients, II in 36 (17.3\%), III in $35(16.8 \%)$, and IV in 11 (5.3\%). The sensitivities of S-p53Ab, CEA, and CA19-9 according to the UICC7/TNM stage were as follows. Among the 126 patients with stage I GC, $13(10.3 \%), 3(2.4 \%)$, and 11 patients $(8.7 \%)$ were positive for S-p53Ab, CA19-9, and CEA, respectively. Among the 36 patients with stage II GC, 9 (25.0\%), 0 (0.0\%), and 12 patients $(33.3 \%)$ were positive for S-p53Ab, CA19-9, and CEA, respectively. The detection sensitivity of a single serum tumor marker was highest for S-p53Ab in stage I GC (Figure 1). We evaluated the benefit of testing for S-p53Ab in addition to measurements of the other two tumor markers. Figure 2 shows the detection sensitivities of S-p53Ab+CEA, CEA+CA19-9, and CA19-9+S-p53Ab according to the UICC7/TNM stage. Among the 126 patients with stage I GC, $23(18.3 \%), 11(8.7 \%)$, and 15 patients $(11.9 \%)$ were positive for S-p53Ab+CEA, CEA+CA19-9, and CA19-9+S-p53Ab, respectively (Figure 2). The detection sensitivities of $S$ p53Ab+CEA, CEA+CA19-9, and CA19-9+S-p53Ab combinations in the 208 patients were $29.3 \%(n=51), 20.2 \%$ $(n=42)$, and $20.7 \%(n=43)$, respectively, with S-p53Ab+CEA as the most sensitive.
Characteristics of patients and their surgical outcomes. Results of univariate analysis of postoperative mortality, using the same factors as those in Table I, are presented Table II. The median and minimum follow-up times for survivors were 34 months and 2 months, respectively. All factors were associated with mortality except for sex, age, tumor site, total number of dissected lymph nodes, lymph node dissection, C-reactive protein, GPS, CEA, and Sp53Ab. The total number of metastatic lymph nodes was the most sensitive predictor of postoperative mortality (hazard ratio $(\mathrm{HR})=13.332 ; 95 \%$ confidence interval $(\mathrm{CI})=6.472$ $27.464 ; p<0.001)$. Multivariate analyses of GPS, CEA, CA19-9, and S-p53Ab revealed that CA19-9 $(\mathrm{HR}=3.864$; 95\% CI=1.248-11.9594; $p=0.019)$ was associated with postoperative mortality (Table II).

Kaplan-Meier analysis demonstrated significant differences between two groups of patients: S-p53Ab(-) or CEA(-) (mean survival, 77.3 months; 95\% confidence interval, 72.6-82.0 months) and S-p53Ab(+) and $\mathrm{CEA}(+)$ (mean survival, 32.7 months; 95\% confidence interval, 21.0-44.3 months) (Figure 3).

\section{Discussion}

The autoantibody $\mathrm{p} 53 \mathrm{Ab}$ is induced by mutation of the $p 53$ tumor suppressor gene and has been detected in the serum of patients with various types of cancer $(3,15-17)$. Several studies have demonstrated that S-p53Ab can serve as an 


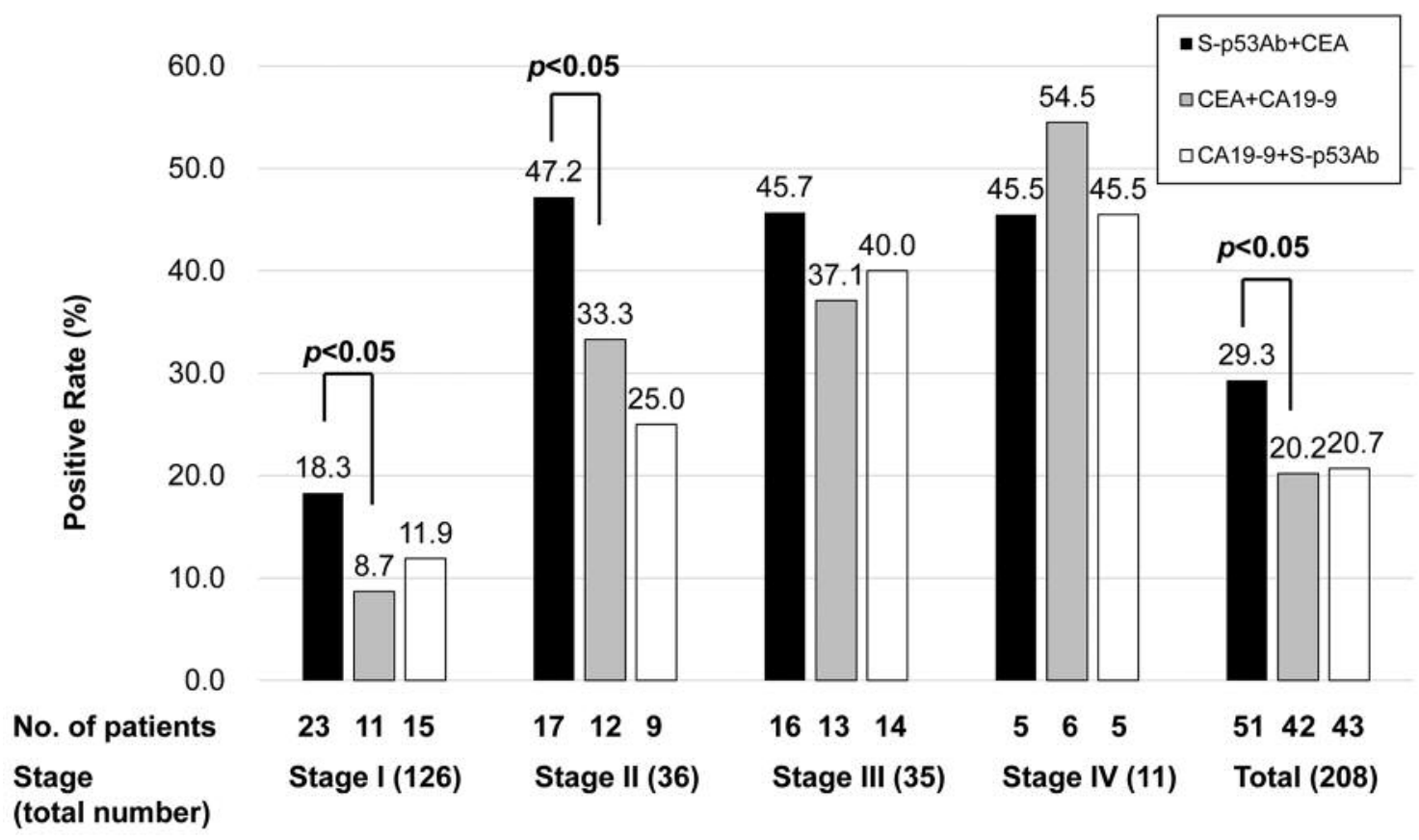

Figure 2. Correlation between clinical stage and sensitivity of biomarker combinations. S-p53Ab, Serum-p53 antibody; CEA, carcinoembryonic antigen; CA19-9, carbohydrate antigen 19-9.

early detection marker of malignant disease, as a marker of treatment effects in patients with malignant tumors including $\mathrm{GC}$, and as a prognostic factor for patients with several types of cancers (18-21).

The Japanese Authority approved the S-p53Ab kit (MBL, Nagoya, Japan) in 2007 for detection of this tumor marker for use in colorectal cancer, breast cancer, and esophageal cancer, but not gastric cancer, with the cost of S-p53 Ab measurement covered by the Health Insurance System. Since then, the S-p53Ab ELISA kit from MBL has become widely used in general medical practice.

GC can be diagnosed at an early stage due to advances in medical technology, and testing potential biological markers for early diagnosis of GC is also important. Although previous studies have evaluated the clinical value of $\mathrm{S}-\mathrm{p} 53 \mathrm{Ab}$ in different conditions, the role of S-p53Ab in patients with GC has not been clearly established. We conducted the present study to evaluate S-p53Ab as a tumor marker of $\mathrm{GC}$, as measured using the S-p53Ab kit in patients who underwent surgery. The present study showed that GC was preoperatively detectable using this S-p53Ab kit in 34 (16.3\%) of 208 patients, which is comparable with previous findings in patients with GC (22). Among 126 patients with stage I GC, 13 $(10.3 \%), 3(2.4 \%)$, and $11(8.7 \%)$ were positive for S-p53Ab, CA19-9, and CEA, respectively. The positive rate of S-p53Ab was significantly higher than that of CA19-9 in stages I and II,

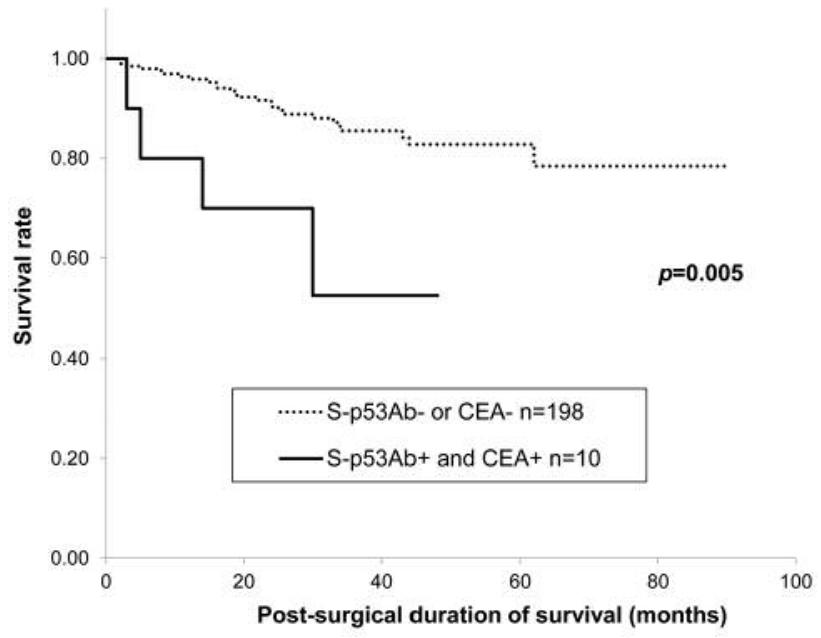

Figure 3. Overall survival among patients with gastric cancer according to positivity for serum 553 antibody $(S-p 53 A b)$ and carcinoembryonic antigen (CEA). Positivity for both markers was associated with significantly reduced survival. S-p53Ab, Serum-p53 antibody; CEA, carcinoembryonic antigen.

suggesting that the S-p53Ab test is more sensitive for detecting cancer at an early stage. On the other hand, the positive rate of S-p53Ab was lower than that for CEA and CA19-9 in stage IV patients, probably because the S-p53Ab test depends on the 
Table II. Univariate and multivariate analyses of survival using Cox proportional hazard models in patients with gastric cancer.

\begin{tabular}{|c|c|c|c|c|c|c|}
\hline \multirow[b]{2}{*}{ Variables } & \multicolumn{3}{|c|}{ Univariate } & \multicolumn{3}{|c|}{ Multivariate } \\
\hline & $p$-Value & $\mathrm{HR}$ & $95 \% \mathrm{CI}$ & $p$-Value & HR & $95 \% \mathrm{CI}$ \\
\hline Gender (male/female) & 0.814 & 0.911 & $0.417-1.989$ & & & \\
\hline Age $<65 / \geq 65$ years & 0.178 & 1.711 & $0.783-3.738$ & & & \\
\hline Tumor site (DLM/UE) & 0.865 & 1.073 & $0.477-2.415$ & & & \\
\hline Histological type (diff/undiff) & 0.044 & 2.184 & $1.022-4.667$ & & & \\
\hline Tumor depth pT1-2/T3-4 & $<0.001$ & 6.970 & $3.097-15.686$ & & & \\
\hline TNM stage 0 -II/III-IV & $<0.001$ & 8.407 & $3.986-17.732$ & & & \\
\hline Lympatic invasion $0 / 1,2,3$ & 0.010 & 3.547 & $1.357-9.274$ & & & \\
\hline Venous invasion $0 / 1,2,3$ & 0.002 & 4.692 & $1.794-12.267$ & & & \\
\hline Nodal stage N0/N1-3 & $<0.001$ & 3.898 & $1.838-8.267$ & & & \\
\hline Total number of metastatic lymph nodes $0-9 / 10-34$ & $<0.001$ & 13.332 & $6.472-27.464$ & & & \\
\hline Total number of dissected lymph nodes 0-39/40-97 & 0.930 & 0.968 & $0.470-1.996$ & & & \\
\hline Lymph node dissection (D) D0,1/D1+,D2 & 0.679 & 0.738 & $0.176-3.101$ & & & \\
\hline Surgery Laparoscopic/Open & 0.002 & 3.158 & $1.537-6.485$ & & & \\
\hline C-reactive protein $<1.0 / \geq 1.0 \mathrm{U} / \mathrm{ml}$ & 0.115 & 2.624 & $0.790-8.713$ & & & \\
\hline Albumin $<3.5 / \geq 3.5 \mathrm{~g} / \mathrm{dl}$ & 0.012 & 0.257 & $0.089-0.741$ & & & \\
\hline GPS $0 / 1,2$ & 0.053 & 2.593 & $0.989-6.795$ & 0.423 & 1.610 & $0.502-5.168$ \\
\hline CEA & 0.248 & 1.648 & $0.706-3.851$ & 0.680 & 0.801 & $0.279-2.299$ \\
\hline CA19-9 & 0.001 & 4.551 & $1.852-11.183$ & 0.019 & 3.864 & $1.248-11.959$ \\
\hline S-p53Ab & 0.056 & 2.206 & $0.979-4.971$ & 0.091 & 2.052 & $0.891-4.726$ \\
\hline
\end{tabular}

D, Deuodenum; LMN, the lower(L), middle (M), and upper(U) parts of stomach; E, esophagus; GPS, Glasgow prognostic score; S-p53Ab, Serum p53 antibody; CEA, carcinoembryonic antigen; CA19-9, carbohydrate antigen 19-9; HR, hazard ratio; 95\% CI, 95\% confidence interval.

immune system's response to mutant p53 protein. In advanced cancers, the period of exposure of the mutant $\mathrm{p} 53$ protein to the immune system is longer than in early-stage cancer. This repeated exposure of the p53 antigen to the immune system may induce immunological tolerance, resulting in a negative $\mathrm{S}$-p53Ab test result (12). These data suggested that detection of S-p53Ab may be related to early carcinogenesis following p53 gene alteration.

Notably, $85.3 \%$ of patients who were positive for S-p53Ab did not have high CA19-9 levels, and only $29.4 \%$ of those positive for S-p53Ab had high CEA levels. The presence of Sp53Ab was not associated with serum CA19-9 or CEA ( $p=0.053$ and $p=0.098$, respectively), and thus, S-p53Ab may serve as a detection marker for GC independent of CA19-9 or CEA. S-p53Ab is more sensitive for detecting various cancers at an early stage than other conventional tumor markers, so Sp53 Ab may useful surveillance modality after GC resection. The positive rate for a diagnosis of GC increased from $16.3 \%$ to $29.3 \%$ when S-p53-Ab was combined with CEA. The positive rate of S-p53Ab+CEA was significantly higher than that of CEA+CA19-9 in stages I and II, suggesting that the combination of S-p53Ab+CEA was a more sensitive biomarkers set for detecting GC at an early stage (Figure 2).

Several studies have identified S-p53Ab as a prognostic factor for patients with various types of tumors, but the prognostic value in patients with GC remains controversial. In this study, the presence of S-p53Ab was not significantly correlated with overall survival, whereas Cox regression analysis revealed that a high level of CA19-9 was an independent prognostic factor in our patients with GC (Table II). Kaplan-Meier analyses demonstrated significant differences in survival between patients with elevated levels of both S-p53Ab and CEA and those with no elevation of these factors or elevation of only one biomarker (Figure 3). The combination of S-p53Ab+CEA was, thus, useful for detecting GC and was able to clearly divide patients with GC into two independent groups, and positivity for S-p53Ab and CEA was associated with poor survival of GC.

This study has several limitations. Firstly, the sample population was small, the follow-up period was short (median 34 months), and patients were from a single institution; these limitations may have affected statistical accuracy. Secondly, an inevitable bias was involved due to the retrospective nature of this study. Therefore, a larger-scale, prospective validation study is needed to confirm these results.

\section{Conclusion}

In conclusion, based on the findings of this study, a combination of S-p53Ab and CEA can serve as a useful biomarker for cancer screening and permit more accurate stratification of patients with GC. This, in turn, should improve clinical decision-making, and perhaps contribute to more rational study design and analysis. 


\section{Funding}

None.

\section{Conflicts of Interest}

No financial or other potential conflicts of interest exist for any of the Authors.

\section{References}

1 Jemal A, Bray F, Center MM, Ferlay J, Ward E and Forman D: Global cancer statistics. CA Cancer J Clin 61(2): 69-90, 2011.

2 Lubin R, Schlichtholz B, Teillaud JL, Garay E, Bussel A and Wild CP: p53 antibodies in patients with various types of cancer: assay, identification and characterization. Clin Cancer Res 1: 1463-1469, 1995.

3 Soussi T, Legros Y, Lubin R, Ork K and Schlichtholz B: Multifactorial analysis of p53 alteration in human cancer: a review. Int J Cancer 57: 1-9, 1994.

4 Change F, Syrjanen S and Syrjanen K: Implication of the p53 tumor suppressor gene in clinical oncology. J Clin Oncol 13: 1009-1022, 1995.

5 The Cancer Genome Atlas Research Network (2011).

6 Shah SP, Roth A, Goya R, Oloumi A, Ha G, Zhao Y, Turashvili G, Ding J, Tse K, Haffari G, Bashashati A, Prentice LM, Khattra J, Burleigh A, Yap D, Bernard V, McPherson A, Shumansky K, Crisan A, Giuliany R, Heravi-Moussavi A, Rosner J, Lai D, Birol I, Varhol R, Tam A, Dhalla N, Zeng T, Ma K, Chan SK, Griffith M, Moradian A, Cheng SW, Morin GB, Watson P, Gelmon K, Chia S, Chin SF, Curtis C, Rueda OM, Pharoah PD, Damaraju S, Mackey J, Hoon K, Harkins T, Tadigotla V, Sigaroudinia M, Gascard P, Tlsty T, Costello JF, Meyer IM, Eaves CJ, Wasserman WW, Jones S, Huntsman D, Hirst M, Caldas C, Marra MA and Aparicio S: The clonal and mutational evolution spectrum of primary triple-negative breast cancers. Nature 486(7403): 395-399, 2012.

7 Rudin CM, Durinck S, Stawiski EW, Poirier JT, Modrusan Z, Shames DS, Bergbower EA, Guan Y, Shin J, Guillory J, Rivers CS, Foo CK, Bhatt D, Stinson J, Gnad F, Haverty PM, Gentleman R, Chaudhuri S, Janakiraman V, Jaiswal BS, Parikh C, Yuan W, Zhang Z, Koeppen H, Wu TD, Stern HM, Yauch RL, Huffman KE, Paskulin DD, IIIei PB, Varella-Garcia M, Gazdar AF, de Sauvage FJ, Bourgon R, Minna JD, Brock MV and Seshagiri S: Comprehensive genomic analysis identifies SOX2 as a frequently amplified gene in small-cell lung cancer. Nat Genet 44(10): 1111-1116, 2012.

8 Yachida S, White CM, Naito Y, Zhong Y, Brosnan JA, Macqregor-Das AM, Morgan RA, Saunders T, Laheru DA, Herman JM, Hruban RH, Klein AP, Jones S, Velculescu V, Wolfgang CL and lacobuzio-Donahue CA: Clinical significance of the genetic landscape of pancreatic cancer and implications for identification of potential long-term survivors. Clin Cancer Res 18(22): 6339-6347, 2012.

9 Masaki Kunizaki, Terumitsu Sawai, Hiroaki Takeshita Tetsuro Tominaga, Shigekazu Hidaka, Kazuo To, Takuro Miyazaki, Ryuji Hamamoto, Atsushi Nanashima and Takeshi Nagayasu: Clinical Value of Serum p53 Antibody in the Diagnosis and Prognosis of Colorectal Cancer. Anti cancer research 36(8): 4171-4175, 2016.
10 von Breveren MC, Hollstein MC, Cawley HM, De Benedetti VM, Bennett WP, Liang L, He AG, Zhu SM, Tursz T, Janin N and Trivers GE: Circulating anti-p53 antibodies in oesophageal cancer patients are found predominantly in individuals with p53 core domain mutations in their tumors. Cancer Res 56: 4917-4921, 1996.

11 Hideaki Shimada, Takenori Ochiai and Fumio Nomura: Titration of serum p53 antibodies in 1085 patients with various types of malignant tumors. Cancer 97(3): 682-689, 2013.

12 Hiroki Ochiai, Takashi Ohishi, Koji Osumi, Jo Tokuyama, Hidejirou Urakami, Shikou Seki, Atsushi Shimada, Akira Matsui, Yoh Isobe, Yuya Murata, Takashi Endo, Yoshiyuki Ishii, Hirotoshi Hasegawa, Sumio Matsumoto and Yuko Kitagawa: Reevaluation of serum p53 antibody as a tumor marker in colorectal cancer patients. Surg Today 42: 164-168, 2012.

13 Sobin LH, Gospondarowicz MK and Wittekind C: TNM classification of malignant tumours. 7th ed. New York: WileyBlackwell; 2009.

14 Ishizuka M, Nagata H, Takagi K, Iwasaki Y and Kubota K: Inflammation-based prognostic system predicts postoperative survival of colorectal cancer patients with a normal preoperative serum level of carcinoembryonic antigen. Ann Surg Oncol 19: 3422-3431, 2012.

15 Kato A, Tsuji T, Sasako Y, Ohashi N, Yasuda H, Fujimoto T, Takita T, Furuhashi $\mathrm{M}$ and Kumagai $\mathrm{H}$ : A comparison of Systemic Inflammation-Based Prognostic Scores in Patients on Regular Hemodialysis. Nephron Extra 3: 91-100, 2013.

16 Angelopoulou K, Diamandis EP, Sutherland DJ, Kellen JA and Bunting PS: Prevalence of serum antibodies against the p53 tumor suppressor gene protein in various cancers. Int J Cancer 58: 480-487, 1994.

17 Mattioni M, Soddu S, Prodosmo A, Visca P, Conti S, Alessandrini G, Facciolo F and Strigari L: Prognostic role of serum p53 antibodies in lung cancer. BMC Cancer 15: 148, 2015.

18 Peyrat JP, Bonneterre J, Lubin R, Vanlemmens L, Fournier J and Soussi T: Prognostic significance of circulating p53 antibodies in patients undergoing surgery for locoregional breast cancer. Lancet 345: 621-622, 1995.

19 Tokunaga K, Sakamoto Y, Nakagawa S, Toshida N and Baba H: The utility of tumor marker combination, including serum p53 antibody, in colorectal cancer treatment. Surg Today, 2017. doi: 10. 1007/s00595-016-1464-8

20 Kawahara D, Fujita F, Hayashi H, Matsuyama T and Eguchi S: The significance of combined measurement of p53 antibody and other tumor markers for colorectal cancer after curative resection. Hepatogastoenterology 62(139): 624-628, 2015.

21 Shimizu K, Ueda Y and Yamagishiazu H: Titration of serum p53 antibodies in patients with gastric cancer: a single-institute of 40 patients. Gastric Cancer 8: 214-219, 2005.

22 Maehara Y, Kakeji Y, Watanabe A, Baba H, Kusumoto H, Kohnoe S and Sugimachi K: Clinical implications of serum antip53 antibodies for patients with gastric carcinoma. Cancer 85(2): 302-308, 1999. 